\title{
Distributional analyses in auditory lexical decision: Neighborhood density and word-frequency effects
}

\author{
Winston D. GoH, Lidia Suárez, Melvin J. YaP, ANd Seok Hui Tan \\ National University of Singapore, Singapore
}

\begin{abstract}
In the present article, the effects of phonological neighborhood density and word frequency in spoken word recognition were examined using distributional analyses of response latencies in auditory lexical decision. A density $X$ frequency interaction was observed in mean latencies; frequency effects were larger for low-density words than for high-density words. Distributional analyses further revealed that for low-density words, frequency effects were reflected in both distributional shifting and skewing, whereas for high-density words, frequency effects were purely mediated by distributional skewing. The results suggest that word frequency plays a role in early auditory word recognition only when there is relatively little competition between similar-sounding words, and that frequency effects in high-density words reflect postlexical checking.
\end{abstract}

Phonological neighborhood density and word frequency produce very robust effects in the auditory lexical decision task (LDT), in which participants discriminate words from nonwords. Phonological neighborhood density refers to how similar a word sounds relative to other words in the mental lexicon, and is defined by the number of neighbors - that is, words differing from the target word by a single phoneme. Reaction time (RT) slows as density increases (Goldinger, Luce, \& Pisoni, 1989; Luce \& Pisoni, 1998; Vitevitch \& Luce, 1999; Ziegler, Muneaux, \& Grainger, 2003), suggesting that lexical representations of similar-sounding words are activated and either compete with (see, e.g., Luce \& Pisoni, 1998) or inhibit (e.g., McClelland \& Elman, 1986; Norris, McQueen, \& Cutler, 2000) each other during recognition. Word-frequency effects, in which latencies are faster for more common words, have also been found in numerous LDT studies, and they are incorporated in models as a bias toward higher frequency words, although the precise locus of this bias differs across different models.

In all auditory LDT studies thus far, mean RTs were used as the dependent variable (DV). The underlying assumption is that RT distributions across experimental conditions are symmetrical, and effects of interest are inferred by whether the mean RTs change across conditions, implicating the shifting of the RT distribution without a change of shape. However, mean differences, or the lack thereof, can also be due to changes in the skew of the distribution, either alone or in combination with a shift in the modal portion of the distribution. Null results can even arise from the shifting and skewing effects canceling each other out (Heathcote, Popiel, \& Mewhort, 1991). Traditional mean
RT analyses may therefore obscure the nature of the effects, and distributional analyses provide a finer grained analytical technique that will allow researchers to pinpoint more precisely the locus of effects (Balota, Yap, Cortese, $\&$ Watson, 2008). For example, word-frequency effects are reflected by both distributional shifting and skewing in the visual LDT, and it has been argued that this implicates frequency influences in both early lexical access (shifting) and late postlexical checking (skewing) processes that are specific to the LDT (Andrews \& Heathcote, 2001; Balota \& Chumbley, 1984; Balota \& Spieler, 1999).

Two techniques, ex-Gaussian and Vincentizing analyses (Balota et al., 2008), were used to examine shifting and skewing in the RT distributions. Ex-Gaussian analyses iteratively search for a set of parameters that maximizes the goodness of fit of raw RT data to an explicit model, the exGaussian distribution. This distribution is a convolution of two underlying distributions, the Gaussian (normal) and exponential distributions. Three parameters describe the distribution; $\mu$ and $\sigma$ are, respectively, the mean and standard deviation of the Gaussian component, and $\tau$ is the mean and standard deviation of the exponential component. One important property of the ex-Gaussian function is that the mean of the RT distribution is the algebraic sum of $\mu$ and $\tau$. This constraint allows the partitioning of mean differences into components than can be separately attributed to distributional shifting $(\mu)$ and skewing $(\tau)$, and thus provides a finer grained analysis as compared with examining mean RT alone. Conceptually, this means that one can determine whether an independent variable (IV) is primarily shifting, skewing, or shifting and skewing a distribution. Traditional mean RT analyses are unable to

W. D.Goh,psygohw@nus.edu.sg 
do this because the mean effect subsumes both shifting and skewing.

Vincentizing (Vincent, 1912) was used to complement the ex-Gaussian analyses. Vincentizing is a nonparametric technique that averages quantiles $(10 \mathrm{th}, 30 \mathrm{th}, 50 \mathrm{th}, 70 \mathrm{th}$, and 90th percentiles) across individual RT distributions within a condition to produce a group RT distribution (Jiang, Rouder, \& Speckman, 2004) for that condition. This provides a useful nonparametric graphical complement to ex-Gaussian analyses, and shows how an IV affects different regions of the RT distribution.

The present study leveraged on these analytical techniques to explore the distributional characteristics of density and frequency effects in auditory word recognition, using the auditory LDT. Given the exploratory nature of this work, the predictions are not entirely clear. For example, although Luce and Pisoni's (1998) neighborhood activation model (NAM) suggests that frequency effects are the result of a bias at the decision stage, it is not sufficiently well specified to make predictions at the distributional level. However, as was discussed earlier, work in the visual word recognition domain indicates that frequency effects are reflected by distributional shifting and skewing, which is consistent with early lexical processes and late postlexical decision processes (see Balota \& Chumbley, 1984). With regard to neighborhood density, no study to our knowledge has investigated the effect of density on RT distributions. However, given that most spoken word recognition models postulate that the recognition of high-density words will be hindered by greater neighborhood activity, density effects should largely reflect early lexical access, which is manifested as a shift in the RT distribution. Finally, turning to the joint effects of frequency and density, Luce and Pisoni reported that frequency effects were larger for low-density words than for high-density words. Although these two factors interact at the level of the mean, it is unclear how the interaction is manifested in the underlying distribution. Exploring this interaction between density and frequency was another objective of the present study.

\section{METHOD}

\section{Participants}

Fifty introductory psychology students from the National University of Singapore, who had no reported history of speech or hearing impairment, were paid $\mathrm{S} \$ 5$ or participated for course credit.

\section{Design and Materials}

A 2 (neighborhood density: low, high) $\times 2$ (word frequency: low, high) within-subjects design was used, with 46 consonant-vowelconsonant (CVC) words for each of the four conditions. Table 1 summarizes the word properties. An $\alpha$ level of .05 was adopted for all analyses, unless otherwise stated. Two-way ANOVAs showed a main effect of frequency $\left[F(1,180)=524.48, M S_{\mathrm{e}}=0.15\right]$ for the log-frequency values (low frequency, $M=0.34, S D=0.28$; high frequency, $M=1.66, S D=0.47)$, and a main effect of density $\left[F(1,180)=869.80, M S_{\mathrm{e}}=8.64\right]$ for the density values (low density, $M=11.75, S D=3.18$; high density, $M=24.53, S D=2.65)$. No other effects were significant $\left(F_{\mathrm{S}}<1\right)$. The conditions were also equated on their average neighborhood frequency and spoken word duration (all $F \mathrm{~s}<1.7$ ). The 184 nonwords were constructed by recombining the onset and rime portions of the $\mathrm{CVC}$ words to create pronounceable words, and they were checked against the ARC nonword database (Rastle, Harrington, \& Coltheart, 2002) to ensure that they were legal English combinations.

All stimuli were spoken by a linguistically trained female Singaporean and were digitally recorded in a 16-bit mono, $44.1-\mathrm{kHz}$ .wav format. The overall root-mean square amplitude levels of the sound files were digitally leveled. Twenty undergraduates from an independent sample identified the stimuli, and tokens that did not achieve at least $70 \%$ accuracy were rerecorded and retested. The mean correct-identification levels for both words and nonwords were $89 \%(S D \mathrm{~s}=9)$. Each word also had an average familiarity rating of 6.2 or higher on a 7-point scale (higher is more familiar) that was based on an unrelated study using the same population, and thus, each was highly familiar to participants.

\section{Procedure}

Participants were tested on individual PCs in groups of 5 or fewer. E-Prime 1.2 and the PST serial response box (Schneider, Eschman, \& Zuccolotto, 2002) were used for stimuli presentation and data collection. Stimuli were binaurally played through Beyerdynamic DT150 headphones at approximately $70 \mathrm{db}$ SPL. Participants indicated as quickly and as accurately as possible whether the token played on each trial was a word or a nonword, using the right- and leftmost buttons on the buttonbox, respectively. RT was measured from the stimulus onset to the buttonpress. After each response, $500 \mathrm{msec}$ elapsed before the next token was played. Practice trials with 20 unrelated stimuli were followed by 368 experimental trials that were randomly presented for each participant, with a short break after 184 trials.

\section{RESULTS}

Errors and latencies ${ }^{1}$ that were faster than 200 msec or slower than 3,000 msec were first excluded, followed by the overall means and $S D$ s for each participant computed across

Table 1

Mean Word Properties and Their Standard Deviations in the Experimental Conditions

\begin{tabular}{|c|c|c|c|c|c|c|c|c|}
\hline \multirow[b]{2}{*}{ Conditions } & \multicolumn{2}{|c|}{ Log Frequency } & \multicolumn{2}{|c|}{ Density } & \multicolumn{2}{|c|}{$\begin{array}{c}\text { Log } \\
\text { Neighborhood } \\
\text { Frequency }\end{array}$} & \multicolumn{2}{|c|}{$\begin{array}{l}\text { Word } \\
\text { Duration } \\
\text { (msec) }\end{array}$} \\
\hline & $M$ & $S D$ & $M$ & $S D$ & $M$ & $S D$ & $M$ & $S D$ \\
\hline \multicolumn{9}{|l|}{ Low Density } \\
\hline Low frequency & 0.34 & 0.34 & 11.67 & 3.23 & 2.00 & 0.39 & 574 & 89 \\
\hline High frequency & 1.67 & 0.51 & 11.85 & 3.18 & 2.07 & 0.28 & 557 & 82 \\
\hline \multicolumn{9}{|l|}{ High Density } \\
\hline Low frequency & 0.34 & 0.22 & 24.39 & 2.43 & 1.99 & 0.21 & 581 & 80 \\
\hline High frequency & 1.66 & 0.44 & 24.67 & 2.88 & 2.10 & 0.23 & 591 & 103 \\
\hline
\end{tabular}

Note-Density and neighborhood frequency scores were obtained from the Washington University Speech and Hearing Lab Neighborhood Database; log frequency is based on the CELEX spoken language frequency counts. 
all conditions, after which latencies exceeding $2.5 S D$ s from the participant's respective mean were removed. Table 2 summarizes the results obtained for mean latencies, accuracy, and the ex-Gaussian parameters. Two-way ANOVAs by participants and items were performed for latencies and accuracy and by participants for the parameters.

For latency, reliable main effects of density $\left[F_{p}(1,49)=\right.$ 93.69, $M S_{\mathrm{e}}=1,298.54 ; F_{i}(1,180)=11.93, M S_{\mathrm{e}}=$ $9,171.23]$ and frequency $\left[F_{p}(1,49)=50.60, M S_{\mathrm{e}}=\right.$ $\left.1,223.54 ; F_{i}(1,180)=7.03, M S_{\mathrm{e}}=9,171.23\right]$ were obtained. These were qualified by a significant interaction by participants $\left[F_{p}(1,49)=17.90, M S_{\mathrm{e}}=1,030.01\right]$; the items interaction approached significance $\left[F_{i}(1,180)=\right.$ 2.77, $\left.M S_{\mathrm{e}}=9,171.23, p=.098\right]$. All four simple main effects were significant $\left[F_{p} \mathrm{~s}(1,49)>6.16, M S_{\mathrm{e}} \mathrm{s}<\right.$ $1,218.35]$; at both density levels, participants were slower in responding to low-frequency than to high-frequency words; at both frequency levels, participants were faster in responding to low-density than to high-density words. However, as is shown in Table 2, the source of the interaction is a smaller frequency effect for high-density $(16 \mathrm{msec})$ than for low-density $(54 \mathrm{msec})$ words.

For accuracy, none of the items analyses were significant [all $F_{i} \mathrm{~s}(1,180)<2.25, M S_{\mathrm{e}} \mathrm{s}=0.009$ ], but for the analyses by participants, there were reliable main effects of density $\left[F_{p}(1,49)=7.88, M S_{\mathrm{e}}=0.002\right]$ and frequency $\left[F_{p}(1,49)=8.64, M S_{\mathrm{e}}=0.003\right]$. These were qualified by the significant interaction $\left[F_{p}(1,49)=5.75, M S_{\mathrm{e}}=\right.$ $0.011]$. As is shown in Table 2, the reason for this interaction is the higher accuracy rate in the low-density, highfrequency condition relative to those in the other three conditions, which is corroborated by the relevant simple effects analyses $\left[F_{p} \mathrm{~s}(1,49)>15.12, M S_{\mathrm{e}} \mathrm{s}=0.00\right]$.

Turning to the ex-Gaussian parameters, for $\mu$, there were reliable main effects of density $[F(1,49)=33.61$, $\left.M S_{\mathrm{e}}=3,650.56\right]$ and frequency $\left[F(1,49)=6.81, M S_{\mathrm{e}}=\right.$ $3,432.84]$. These were qualified by the significant interaction $\left[F(1,49)=5.58, M S_{\mathrm{e}}=2,854.11\right]$. Simple effects of frequency at each density level showed that for lowdensity words, $\mu$ was larger for low-frequency than for high-frequency words $\left[F(1,49)=12.83, M S_{\mathrm{e}}=3,033.93\right]$, but there was no frequency difference for high-density words $(F<1)$. This finding implicates a significant shift in the modal portion of the RT distribution as a function of frequency, but only for low-density words. Simple effects of density at each frequency level showed that $\mu$ was larger for high-density words than for low-density words for both low-frequency $\left[F(1,49)=8.96, M S_{\mathrm{e}}=2,803.26\right]$ and high-frequency $\left[F(1,49)=30.67, M S_{\mathrm{e}}=3,701.42\right]$ words. This result suggests that distributional shifting occurred for both low- and high-frequency words as a function of density.

For $\sigma$, a significant main effect of density was found $\left[F(1,49)=15.82, M S_{\mathrm{e}}=1,167.51\right] . \sigma$ was larger for highdensity $(M=117, S D=38)$ than for low-density $(M=$ $98, S D=38$ ) words. No other effects were significant.

For $\tau$, the main effect of frequency approached significance $\left[F(1,49)=2.81, M S_{\mathrm{e}}=3,350.68, p=.10\right] . \tau$ was larger for low-frequency $(M=173, S D=67)$ than for high-frequency $(M=159, S D=69)$ words. No other effects were significant $\left(F_{\mathrm{S}}<1\right)$. This finding suggests that the frequency effect is mediated by distributional skewing for both low- and high-density words.

Although the $\tau$ main effect did not reach the traditional significance level, recall that in ex-Gaussian analyses, the mean of the RT distribution is the algebraic sum of $\mu$ and $\tau$. The density $\times$ frequency interaction observed in the traditional mean latency analysis is due to a larger frequency effect for low-density $(54 \mathrm{msec})$ than for high-density $(16 \mathrm{msec})$ words, but both simple effects of frequency were still statistically reliable. Analyses of the $\mu$ parameter as a function of word frequency indicate that there is distributional shifting for the low-density $(39 \mathrm{msec})$ but not for the high-density (4 msec) words. Therefore, most of the frequency effect in high-density words must be because of the $\tau$ parameter. From Table 2, the magnitude of the $\tau$ parameter across both low-density $(15 \mathrm{msec})$ and high-density $(12 \mathrm{msec})$ words as a function of frequency suggests a similar extent of distributional skewing, which supports the marginal main effect of frequency with no other reliable effects. Collectively, these findings strongly suggest that the frequency effect observed for high-density words in the traditional mean latency analyses is primarily mediated by distributional skewing, whereas the frequency effect for low-density words is mediated by both distributional shifting and skewing.

Table 2

Mean Latencies (in Milliseconds), Accuracy (\% Correct), and Ex-Gaussian Parameter Estimates and Their Standard Deviations Across Neighborhood Density and Word Frequency

\begin{tabular}{|c|c|c|c|c|c|c|c|c|c|c|}
\hline \multirow[b]{2}{*}{ Conditions } & \multicolumn{2}{|c|}{ Latency } & \multicolumn{2}{|c|}{ Accuracy } & \multicolumn{2}{|c|}{$\mu$} & \multicolumn{2}{|c|}{$\sigma$} & \multicolumn{2}{|c|}{$\tau$} \\
\hline & $M$ & $S D$ & $M$ & $S D$ & $M$ & $S D$ & $M$ & $S D$ & $M$ & $S D$ \\
\hline \multicolumn{11}{|l|}{ Low Density } \\
\hline Low frequency & 946 & 108 & 88 & 7 & 774 & 82 & 103 & 53 & 174 & 68 \\
\hline High $\mathrm{f}$ & 892 & 99 & 92 & 6 & 735 & 77 & 93 & 43 & 159 & 84 \\
\hline Frequency effect & 54 & & -4 & & 39 & & 10 & & 15 & \\
\hline \multicolumn{11}{|l|}{ High Density } \\
\hline Low frequency & 976 & 110 & 88 & 9 & 806 & 93 & 115 & 48 & 172 & 87 \\
\hline High frequency & 960 & 101 & 88 & 7 & 802 & 96 & 119 & 42 & 160 & 83 \\
\hline Frequency effect & 16 & & 0 & & 4 & & -4 & & 12 & \\
\hline Interaction & 38 & & -4 & & 35 & & 14 & & 3 & \\
\hline Nonwords & 1,055 & 162 & 81 & 14 & 882 & 137 & 124 & 62 & 173 & 76 \\
\hline
\end{tabular}


To examine this suggestion further, quantile analyses were performed on the RT data. Figure 1 shows the mean quantiles across the different conditions. The standard error bars give an indication of the range of the empirical mean quantiles - that is, the mean RTs across participants for each of the five quantiles - whereas the lines represent the theoretical quantiles of the best-fitting ex-Gaussian distribution. This graphical presentation allows a visual assessment of the goodness of fit between the empirical and estimated quantiles. In Figure 1, it is quite clear that the ex-Gaussian distribution fits the empirical data well, since discrepancies between the theoretical and empirical quantiles are within a standard error of the latter.

In the top panel, it can be clearly seen that the frequency effect is observed for low-density words across all quantiles. High-frequency means are always below lowfrequency means in each quantile. In the middle panel, the frequency effect is apparent only at the last quantile. The differential frequency effects can be more clearly seen in the bottom panel, which plots the difference scores between the low- and high-frequency means for the low- and high-density conditions. For the low-density condition, the difference between low- and high-frequency words becomes larger as the RT increases, indicating that the frequency effect is mediated by distributional shifting and skewing. In contrast, for high-density words, the frequency effect is reflected mainly in the slowest RTs, indicating distributional skewing.

\section{DISCUSSION}

RT distributional analyses of phonological neighborhood density and word frequency effects in auditory lexical decision have not been done in previous studies, which have relied on mean RTs as the primary DV. The findings in the present study can be summarized as follows.

First, consistent with the results of earlier work, highdensity words elicited slower RTs than did low-density words, whereas high-frequency words elicited faster RTs than did low-frequency words.

Second, the frequency effect for low-density words was larger than that for high-density words. This interaction essentially replicated the pattern observed by Luce and Pisoni (1998), who also found a larger frequency effect for low-density words. It should be noted that they did not find a density effect for their low-frequency words, whereas this effect was reliable in the present study. However, the direction of the effect is similar across both studies, because the density effect for high-frequency words $(68 \mathrm{msec})$ was larger than that for low-frequency words (30 msec).

Third, and most important, our distributional analyses revealed that the density $\times$ frequency interaction was primarily the result of differential shifting and skewing of the latency distribution between high- and low-frequency words as a function of density. For low-density words, frequency effects were reflected in both distributional shifting and skewing. For high-density words, frequency effects were purely mediated by distributional skewing. Density effects appear to be purely mediated by distribu-

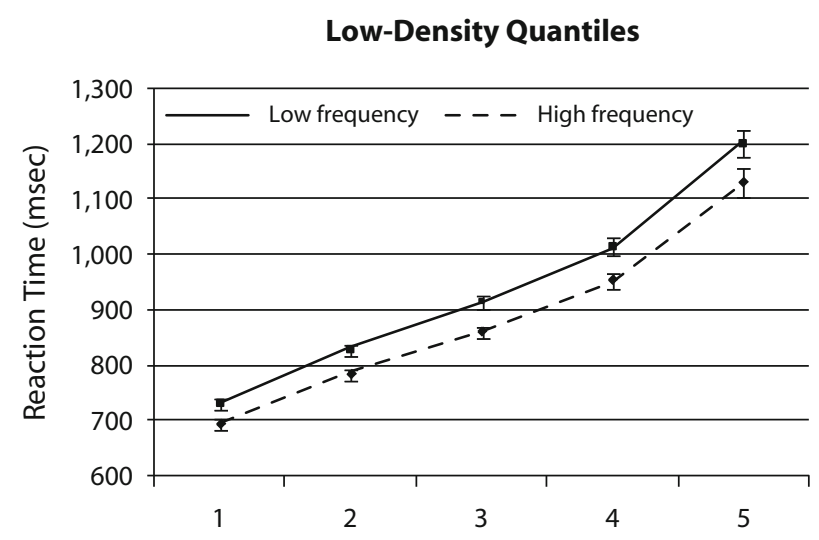

High-Density Quantiles

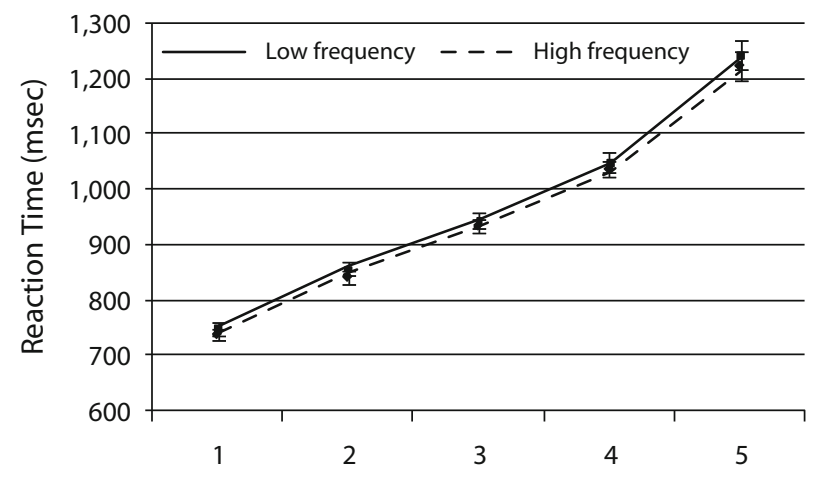

Frequency Effect Quantiles

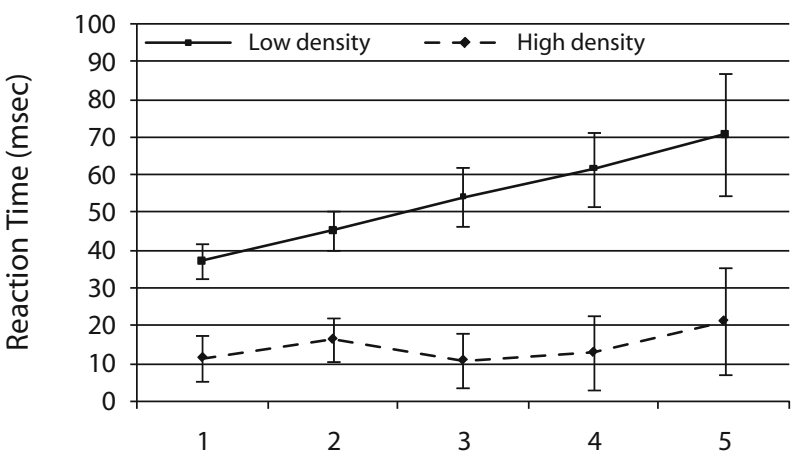

Figure 1. Quantiles (.1, .3, .5, .7, .9) of lexical decision performance. The participants' mean quantiles are represented by data points and standard errors. The lines represent the best-fitting ex-Gaussian quantiles. The top and middle panels show performance as a function of word frequency in the low-density and high-density conditions, respectively, and the bottom panel shows the frequency effect.

tional shifting, since there were effects of density only on the $\mu$ but not on the $\tau$ parameter.

A simple shift in the RT distribution as a function of density is compatible with current accounts of lexical competition among similar-sounding words during spoken word recognition. When there is a higher level of activity because of an increase in the number of compet- 
ing words, more time is needed to resolve the confusion and to get the target word to reach recognition threshold (Luce \& Pisoni, 1998). This increased time to make a response appears to be an additive effect as revealed by the pure distributional shift. Balota and Spieler (1999) have argued that the pure shift effect observed in visual speeded naming tasks is indicative of early automatic processes rather than of later analytical or more attentiondemanding processing. Our present finding that density effects are mediated purely by distributional shifting supports the idea that neighborhood density effects reflect processes that are involved in early lexical access rather than in late postlexical processes implicated in the LDT (see Balota \& Chumbley, 1984).

The interaction between frequency and density, whereby frequency effects are stronger for low-density targets, is more complicated. In NAM, frequency effects are modeled as a bias toward higher level lexical information such as frequency, which increases the activation level of the acoustic-phonetic patterns in the word decision units, allowing high-frequency words to reach the recognition threshold faster. The distributional effects in the present study suggest that this may be true only when there is relatively little competition between similar-sounding words. The frequency effect increases across the RT distribution, consistent with the visual lexical decision literature (e.g., Andrews \& Heathcote, 2001; Balota \& Spieler, 1999), but this effect is limited to low-density words. For high-density words, it appears that there is no effect of frequency except at the slowest end of the distribution, which is reflected in greater skewing for low-frequency words. This result suggests that any early biasing advantage of frequency due to an increased "head start" in reaching the recognition threshold may be offset by increased acoustic-phonetic confusability. The emergence of frequency effects at the tail end of the distribution may reflect postlexical checking processes that are specific to the lexical decision task (see Balota \& Chumbley, 1984), rather than to lexical access per se.

In summary, we replicated the detrimental effects of density and the facilitatory effects of frequency found in earlier studies on auditory lexical decision by using mean RTs as the DV. However, the present study breaks new ground in demonstrating that these effects were differentially reflected in the shift and shape of the underlying RT distributions. Clearly, the greater resolution afforded by finer grained RT distributional analyses has provided additional insight into the possible loci of frequency and density effects with reference to specific models of word recognition and lexical decision performance. In particular, the pure shift effects of density may implicate early lexical processes that are involved in resolving the acoustic-phonetic patterns of the spoken utterance among possible candidates, whereas a combination of shift and skew for frequency suggests both early lexical access and late postlexical decision processes. It is critical for us to note that these differential effects are not observable in traditional mean RT analyses in which the mean subsumes effects of shifting and skewing.

\section{AUTHOR NOTE}

The present work was supported by Research Grant R-581-000-048112 to W.D.G. We thank Weiying Hu for data collection assistance; we also thank Steve Goldinger, Mike Cortese, and two reviewers for constructive comments on an earlier draft. Address correspondence to W. D. Goh, Department of Psychology, National University of Singapore, Singapore 117570 (e-mail: psygohw@nus.edu.sg).

\section{REFERENCES}

Andrews, S., \& Heathcote, A. (2001). Distinguishing common and task-specific processes in word identification: A matter of some moment. Journal of Experimental Psychology: Learning, Memory, \& Cognition, 27, 514-544.

Balota, D. A., \& Chumbley, J. I. (1984). Are lexical decisions a good measure of lexical access? The role of word frequency in the neglected decision stage. Journal of Experimental Psychology: Human Perception \& Performance, 10, 340-357.

Balota, D. A., \& Sieler, D. H. (1999). Word frequency, repetition, and lexicality effects in word recognition tasks: Beyond measures of central tendency. Journal of Experimental Psychology: General, 128, 32-55.

Balota, D. A., Yap, M. J., Cortese, M. J., \& Watson, J. M. (2008). Beyond mean response latency: Response time distributional analyses of semantic priming. Journal of Memory \& Language, 59, 495-523.

Goldinger, S. D., Luce, P. A., \& Pisoni, D. B. (1989). Priming lexical neighbors of spoken words: Effects of competition and inhibition. Journal of Memory \& Language, 28, 501-518.

Heathcote, A., Popiel, S. J., \& Mewhort, D. J. K. (1991). Analysis of response time distributions: An example using the Stroop task Psychological Bulletin, 109, 340-347.

Jiang, Y., Rouder, J. N., \& SPeCKMAN, P. L. (2004). A note on the sampling properties of the Vincentizing (quantile averaging) procedure. Journal of Mathematical Psychology, 48, 186-195.

LucE, P. A., \& Pisoni, D. B. (1998). Recognizing spoken words: The neighborhood activation model. Ear \& Hearing, 19, 1-36.

McClelland, J. L., \& Elman, J. L. (1986). The TRACE model of speech perception. Cognitive Psychology, 19, 1-86.

Norris, D., McQueen, J. M., \& Cutler, A. (2000). Merging information in speech recognition: Feedback is never necessary. Behavioral \& Brain Sciences, 23, 299-325.

Rastle, K., Harrington, J., \& Coltheart, M. (2002). 358,534 nonwords: The ARC Nonword Database. Quarterly Journal of Experimental Psychology, 55A, 1339-1362.

Schneider, W., Eschman, A., \& Zuccolotto, A. (2002). E-Prime user's guide. Pittsburg: Psychology Software Tools, Inc.

VINCENT, S. B. (1912). The function of vibrissae in the behavior of the white rat. Behavioral Monographs, $\mathbf{1}$ (Whole No. 5).

Vitevitch, M. S., \& Luce, P. A. (1999). Probabilistic phonotactics and neighborhood activation in spoken word recognition. Journal of Memory \& Language, 40, 374-408.

Ziegler, J. C., Muneaux, M., \& Grainger, J. (2003). Neighborhood effects in auditory word recognition: Phonological competition and orthographic facilitation. Journal of Memory \& Language, 48, 779-793.

\section{NOTE}

1. Most tokens were edited so that the spoken word's onset corresponded with the sound file's onset. However, approximately $25 \%$ of the tokens had variable amounts of silence at the file's onset, averaging 7-20 msec across conditions. Since E-Prime measures latency from the file onset, we measured the individual onset-silence duration for the affected tokens and subtracted them from the recorded RTs to obtain the proper lexical decision RT for these trials. These corrected values were used in all subsequent analyses. The word duration values in Table 1 reflect the spoken word duration, not the file duration. 
APPENDIX

Words and Nonwords Used in the Experiment

\begin{tabular}{|c|c|c|c|c|c|c|c|c|c|c|c|}
\hline \multicolumn{12}{|l|}{ Words } \\
\hline \multicolumn{12}{|c|}{ Low Frequency, Low Density } \\
\hline boil & chef & dive & germ & hood & jog & mime & noun & tease & vase & web & yawn \\
\hline burp & coin & dove & gown & hook & jug & mop & pawn & toil & verge & wedge & zeal \\
\hline bush & dash & foam & gum & hoop & leash & moth & shawl & toss & vet & womb & \\
\hline cage & dish & gang & hog & jade & lung & nab & tab & tube & wag & worm & \\
\hline \multicolumn{12}{|c|}{ Low Frequency, High Density } \\
\hline beak & chick & dock & ham & kneel & lash & loot & peep & rag & reap & rope & sock \\
\hline bean & chill & dome & heal & lace & lease & mat & pill & raid & reek & sap & tile \\
\hline bone & cock & doom & hip & lake & lice & moan & poke & rake & rip & seal & \\
\hline boot & dine & hack & hug & lap & lick & patch & rack & rave & roam & $\operatorname{sip}$ & \\
\hline \multicolumn{12}{|c|}{ High Frequency, Low Density } \\
\hline beg & cup & foot & jazz & leg & myth & page & roof & soil & thing & vote & wish \\
\hline boss & $\operatorname{dog}$ & fuss & joke & live & name & pub & rush & song & use & walk & wood \\
\hline bus & duke & gas & king & love & noise & put & shop & tape & van & watch & \\
\hline cook & fish & hive & kiss & mood & nose & rob & size & teach & verb & wing & \\
\hline \multicolumn{12}{|c|}{ High Frequency, High Density } \\
\hline beat & call & dean & head & kill & make & note & pick & pope & road & scene & wait \\
\hline bell & cane & fight & heat & light & $\operatorname{man}$ & pack & pile & pot & root & seat & win \\
\hline boat & code & fill & hit & load & meal & pan & pitch & rail & run & sheet & \\
\hline bud & cut & gain & kick & luck & mile & phone & pop & rain & sake & sign & \\
\hline \multicolumn{12}{|c|}{ Nonwords } \\
\hline bæp & ku:f & fi:t $\int$ & $\mathrm{f} \Lambda \int$ & gəup & dzi: $\int$ & leil & nein & poil & su:f & vin & $\operatorname{jed}_{3}$ \\
\hline bi: $\int$ & $\mathrm{k} \wedge \mathrm{g}$ & fi:p & gerk & $\mathrm{g} \Lambda \mathrm{k}$ & dzi:k & lerp & næt $\int$ & pu:m & terd 3 & VIJ & jais \\
\hline beb & $k \wedge \eta$ & fi:k & gæt $\int$ & $g \Lambda f$ & dzi: 1 & lel & nef & pu: $\theta$ & ta: $z$ & vəun & jait \\
\hline bed $_{3}$ & $\mathrm{k} \wedge \int$ & feg & gi:t $\int$ & g3:p & dzais & lorl & nen & po $\theta$ & ti: $\int$ & vok & jain \\
\hline b3:m & derb & fз:b & gi: $\int$ & heIb & dzart & lol & naim & reb & $\theta æ m$ & voIn & jəul \\
\hline bəum & $\operatorname{ded}_{3}$ & fars & gain & hi: $\int$ & dzaim & $\ln \mathrm{n}$ & nIn & rel & Өегр & vวy & jəuz \\
\hline bəub & doIn & faim & g3:m & heb & $\mathrm{d}_{3} \mathrm{It} \int$ & $\operatorname{lnt} \int$ & noIn & roIz & $\theta \mathrm{a}: 1$ & vu:p & jof \\
\hline bəup & doIz & forz & gis & hi:f & dzout & meip & nюy & rju:k & $\theta \mathrm{eg}$ & vวuz & jo $\theta$ \\
\hline$b \Lambda b$ & dəuk & fu:f & git $\int$ & hi:k & dzu:f & meiv & nu:m & seip & $\theta \mathrm{rp}$ & werp & \\
\hline$t \int e r b$ & da:y & fu:m & gəum & hout & dzu:m & $\mathrm{mæz}$ & nu:p & si: $\int$ & $\operatorname{tr} \int$ & wef & \\
\hline t $\int e_{1}$ & du:f & fu:p & gok & holl & kæk & $\operatorname{med} 3$ & nu: $\theta$ & seb & toin & wəut & \\
\hline $\mathrm{t} \int \mathrm{p}: 1$ & duk & fu: $\theta$ & gorl & hoIn & kæy & mef & $\mathrm{n} \Lambda \mathrm{v}$ & sef & tu:p & wəup & \\
\hline 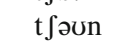 & $\mathrm{du}: \theta$ & fəup & goIz & hi $\theta$ & ki:f & mip & $\mathrm{n} \Lambda \int$ & sIJ & $\operatorname{to} \theta$ & wว $\theta$ & \\
\hline$t \int 0 t$ & $\mathrm{dus}$ & fəuz & gəuk & dzerb & kaIs & mu:p & ni $\theta$ & sit $\int$ & væb & jern & \\
\hline$t \int j u: n$ & ferp & $\mathrm{f} \Lambda \mathrm{V}$ & gu:p & dzerdz & leib & neid 3 & peb & sout & vi:n & ji:t & \\
\hline kગIZ & fæz & $f_{\Lambda} \mathrm{p}$ & gop & dzeiv & $\mathrm{leId}_{3}$ & neIt & parv & soIz & vin & ji:k & \\
\hline
\end{tabular}

(Manuscript received October 9, 2008;

revision accepted for publication May 29, 2009.) 